\title{
The Effect of Progressive Aerobic Exercise On G6PD Activity Among Active and Sedentary Men
}

\author{
Amin Allah Dashtiyan \\ Department of Sport Physiology, University of Mohaghegh Ardebili \\ Ardebil, Iran \\ E-mail: amin.dashtiyan@yahoo.com \\ Marefat Siahkouhian \\ Department of Sport Physiology, University of Mohaghegh Ardebili \\ Ardebil, Iran \\ E-mail: m siahkohian@uma.ac.ir \\ Masoud Ganji \\ Departments of Mathematics and Statistics, University of Mohaghegh Ardebili \\ Ardebil, Iran \\ E-mail: mganji@uma.ac.ir \\ Aidin Valizadeh Oranj \\ Department of Sport Physiology, University of Mohaghegh Ardebili \\ Ardebil, Iran \\ E-mail: valizadeh@uma.ac.ir \\ Hadi Bashafaat (Corresponding author) \\ Department of Sport Physiology, Faculty of General Department of Fars Province Education \\ Fars, Iran \\ E-mail: bashafaat@yahoo.com
}

Received: 26-08- 2014

doi:10.7575/aiac.ijkss.v.2n.4p.7
Accepted: 18-10- 2014

Published: 31-10- 2014

\begin{abstract}
Background: Erythrocyte glucose-6-phosphate dehydrogenase (G6PD) activity is highly associated with free radical production. G6PD deficiency can increase the sensitivity of erythrocytes to oxidative stress resulting in hemolytic anemia. Aim: to study the main effect of progressive aerobic exercise on G6PD activity in active and sedentary men. Material and Methods: the study comprised 10 active men and 10 sedentary men. The protocol, started with running at approximately $\% 75$ of their maximal oxygen uptake for $30 \mathrm{~min} x$ times a week for y weeks. Venous blood samples $(5 \mathrm{ml})$ were collected prior to, immediately after, 2 hours and 24 hours after exercise. G6PD activity was evaluated with auto-Analyzer Method. Result: G6PD was not significantly higher in the active men in comparison with the sedentary men at baseline $(10.5 \pm 1.2(\mathrm{IU} / \mathrm{gHb})$ VS $9.5 \pm 1.0(\mathrm{IU} / \mathrm{gHb}), \mathrm{P} \leq 0.05)$. G6PD activity was increased significantly in both groups immediately after exercise but was not considerably different between the groups $(11.6 \pm 2.7$ (IU/gHb) VS $9.9 \pm 1.1(\mathrm{IU} / \mathrm{gHb})$, for active and sedentary men, respectively; $\mathrm{P} \leq 0.05)$. G6PD returned to the baseline levels 2 hours after exercise in active men but remained high in sedentary men $(10.5 \pm 1.4(\mathrm{IU} / \mathrm{gHb}) \mathrm{VS} 10.1 \pm 1.1(\mathrm{IU} / \mathrm{gHb}, \mathrm{P} \leq 0.05)$. Also, G6PD levels showed a significant increase 24 hours after exercise in the active men in comparison with the sedentary men $(11.8 \pm 2.5$ (IU/gHb) VS $9.5 \pm 1.5$ (IU/gHb), $\mathrm{P} \leq 0.05)$. Conclusion: In this regard, it can be concluded that, progressive aerobic exercise may be an effective factor affecting the levels of G6PD significantly, and as a home message it is useful for controlling the hemolytic anemia among sedentary population.
\end{abstract}

Keywords: G6PD activity, progressive aerobic exercise, hemolytic anemia

\section{Introduction}

Glucose-6-phosphatedehydrogenase (G6PD) is a crucial enzyme in pentose phosphate pathway, because it provides nucleotide precursors and NADPH for the cell (Wamelink, Struys, \& Jakobs, 2008). Glucose-6-phosphate dehydrogenase (G6PD) deficiency is considered a prevalent genetic disorder (it affects roughly 7\% of world population) that can lead to increased sensitivity of erythrocytes to oxidative stress resulting in hemolytic anemia (Allahverdiyev et al., 2012; Nikolaidis et al., 2006). Its major function is reducing nicotinamide adenine dinucleotide phosphate (NADP) to NADPH (Bommareddy, Chen, Rappert, \& Zeng, 2014; Petrat, Pindiur, Kirsch, \& de Groot, 2003). NADPH is necessary as a cofactor in reducing oxidized glutathione (GSSG) to its reduced condition (GSH) and for reducing mixed disulfides of glutathione and cellular proteins (Masella, Di Benedetto, Varì, Filesi, \& Giovannini, 2005; Schafer \& Buettner, 2001). GSH serves as protection against oxidative attack. It is used by the Selenium-Glutathione Peroxidase 
(Se-GSH-Px) system for detoxifying hydrogen peroxide and organic peroxides, as long as enough GSH is available (Nazıroğlu, 2009) (Fig.1).

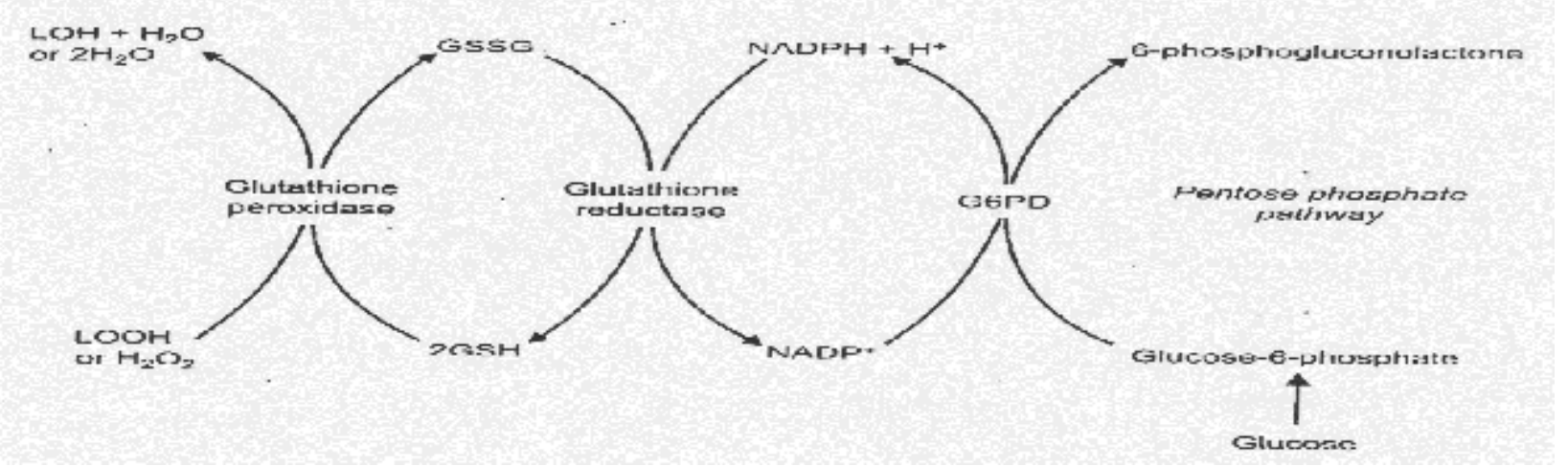

Figure 1. Pentose phosphate pathway (Nikolaidis et al., 2006).

In fact, the reason for the acute hemolytic anemia prompted by hydrogen peroxidase and organic peroxidase is that G6PD deficient erythrocytes are not able to sustain normal levels of GSH (Jamurtas et al., 2006). It is well-known that daily physical exercise and sport performance are helpful. Yet, it has been proved that physical exercise enhances the generation of reactive oxygen species (ROS) in exercising muscles (Niess \& Simon, 2007; Sahlin et al., 2010). In case the production of free radicals is sufficient for overcoming antioxidant defense systems, oxidative stress will occur. Heightened levels of oxidative damage end products are observed in blood and tissues following intense exercise. This seems to be incompatible with the useful effects of exercise (Bloomer, Goldfarb, Wideman, Mckenzie, \& Consitt, 2005; Fisher-Wellman \& Bloomer, 2009).

During exercise, if the generation of ROS increases; erythrocytes will be at a higher risk of oxidative damage (Oztasan et al., 2004).

Cells of G6PD-deficient persons, as a result of their lower levels of GSH and lower potential ability to transform GSSG into GSH, fall short of one of the major radical scavenger molecules against oxidative stress at the time of exercise. Provided that the only way GSH can be recycled is through the pentose phosphate pathway, a crucial step is to catalyze it by erythrocytes and the G6PD enzyme (and other cells to a lesser degree) from G6PD-deficient persons may have defective ability to eliminate lipid peroxides and hydrogen peroxides. The mentioned compounds can trigger oxidation of sulfhydryl groups in proteins as well as peroxidation of lipids in membrane (i.e., hemolysis). If some of the sulfhydryl groups of hemoglobin are oxidized, the protein precipitates within the erythrocyte and forms Heinz bodies (Allahverdiyev et al., 2012; Nikolaidis et al., 2006).

The aim of the present study was to determine the effect of progressive aerobic exercise on G6PD activity in active and sedentary men.

\section{Materials and Methods}

\subsection{Subjects}

Ten men $(n=10)$ with established activity and ten sedentary men with non-activity $(n=10)$ (active subjects were separated from sedentary subjects with maximal oxygen consumption; $\mathrm{VO}_{2 \max }$ ) participated in the present study. Active men practiced for two hours every day. Sedentary men did not do any physical activity during the day. A written informed consent to take part in the research was provided by each of the participants after they were informed of all risks, discomforts, and benefits of the study. All experimental procedures were approved by University of Mohaghegh Ardabili.

\subsection{Anthropometric measurements}

Subjects in any group reported to the laboratory twice. Each subject reported to the lab in the morning after at least 9 hours of fasting and abstaining from alcohol and caffeine for $48 \mathrm{~h}$. In the first visit, the participants' body mass was measured to the nearest $0.5 \mathrm{~kg}$ (Seca, Model 220, Germany). Subjects were lightly dressed and were not wearing shoes. Standing height $(\mathrm{cm})$ was measured with a $0.1 \mathrm{~cm}$ precision by using a stadiometer (Seca, Mode 1220, Germany). Percentage body fat was estimated from three skinfold measures (average of 3 measurements of each site), using a Harpenden caliper (John Bull, UK), according to Jackson and Pollok equation (Nikolaidis et al., 2006). The anthropometric the subjects' characteristics are presented in Table 1.

Table 1.Characteristics of active and sedentary men (mean \pm SD)

\begin{tabular}{lll}
\hline & Sedentary men (A) & Active men (B) \\
\hline Age (yr) & $19.5 \pm .08$ & $20.2 \pm 1.5$ \\
Weight $(\mathrm{kg})$ & $72.0 \pm 12.3$ & $66.3 \pm 6.8$ \\
Height $(\mathrm{cm})$ & $176.2 \pm 7.4$ & $175.0 \pm 3.6$ \\
BMI $\left(\mathrm{kg} / \mathrm{m}^{2}\right)$ & $23.2 \pm 3.7$ & $21.6 \pm 1.9$ \\
Body fat $(\%)$ & $8.8 \pm 5.8$ & $5.7 \pm 2.3$ \\
$V_{\text {o }}$ max $\left(m l . \mathrm{mg}^{-1} \mathrm{~min}^{-1}\right)$ & $42.0 \pm 5.0$ & $58.5 \pm 5.1$ \\
\end{tabular}




\subsection{Short-duration exercise protocol}

To confirm that subjects of active and sedentary groups did not have similar fitness levels, $\mathrm{VO}_{2 \max }$ was measured by using a treadmill test to exhaustion. Modified Bruce protocol on a treadmill (Technogym, Italy) was used to achieve $\mathrm{VO}_{2 \max }$ test in volunteers (Nikolaidis et al., 2006). Subjects' $\mathrm{VO}_{2 \max }$ is shown in Table 1. Henceforth, this $\mathrm{VO}_{2 \max }$ test is referred to as short-duration exercise protocol.

\subsection{Long-duration exercise protocol}

Seven days after the short-duration exercise protocol, for a second time, the subjects visited the lab to perform a single 30 min bout aerobic exercise at approximately $75 \%$ of their maximal oxygen uptake. Speed alterations were made during exercise to ensure that the participants were exercising within $75 \% \mathrm{VO}_{2 \max }$ (Nikolaidis et al., 2006).

\subsection{Dietary condition}

In order to control the impact of previous diet on the outcome measures of the research, and confirm that subjects in both groups had similar macro and micro nutrients intake levels, their food was controlled by a dietarian before and after the study. Meanwhile, they were instructed to follow a normal pattern of eating containing $55 \% \mathrm{CHO}, 20 \%$ Pro, 25 $\%$ Fat and around 8 Lit water daily without any affective supplements (Shariat, Kargarfard, Tamrin, Danaee, \& Karimi, 2014).

\subsection{Evaluation of G6PD activity}

Before long-duration exercise protocol, a blood sample $(5 \mathrm{ml})$ was obtained from a forearm vein. Then, blood samples were collected immediately after, 2 hours and 24 hours after exercise. After that, blood samples were poured into a tube which contained ethylenediamine tetra-acetic acid and to determine G6PD activity were placed immediately at $4^{\circ} \mathrm{C}$. Next, fifty micro liter of the sample was rinsed with serum physiology and after that, one micro liter of the lysis solution was added and placed in a refrigerator at $4^{\circ} \mathrm{C}$ for $5 \mathrm{~min}$. Then, hemolyzed blood samples were centrifuged for $5 \mathrm{~min}$. The supernatants were used for determination of G6PD activity. Finally, samples were placed within auto analyzer (Hitachi Autoanalyser) to determine G6PD activity (Ainoon et al., 2003; Huang et al., 2005). The following formula was used to calculate enzyme activity.

\section{G6PD activity $(\mathrm{IU} / \mathrm{gHb})=\frac{\text { activity of the enzyne (reading from the instrument: }}{\text { Hem }}$ \\ 2.7 Statistical analysis}

Data are shown as mean \pm SD. The distribution of all dependent variables was studied by using the KolmogorovSmirnov test and it was found that it did not significantly different from normal. Differences in physical characteristics and G6PD activity between the active and sedentary groups were examined by independent sample t-test. Repeated measures also were utilized for measuring G6PD activity changes in different times within each group. If an important main effect was obtained, Bonferroni method of adjustment was conducted through analyzing simple main effect. Statistical significance level was set at $\alpha=0.05$. For all analyses, the SPSS version 19.0 was used (SPSS Inc.).

\section{Results}

As table 2 shows, there are no considerable differences between the two groups in any of the physical characteristics ( $\mathrm{P}$ $\leq 0.05$ ). As table 3 shows, G6PD was not considerably higher in the active men compared to the sedentary men at baseline $(10.5 \pm 1.2(\mathrm{IU} / \mathrm{gHb}) \mathrm{VS} 9.5 \pm 1.0(\mathrm{IU} / \mathrm{gHb}))$.

G6PD activity was found to have increased in both groups immediately after long-duration exercise but was not significantly different between the groups $(11.6 \pm 2.7$ (IU/gHb) VS $9.9 \pm 1.1$ (IU/gHb), for active and sedentary men, respectively; $\mathrm{P} \leq 0.05)$. G6PD returned to the baseline levels 2 hours after long-duration exercise in active men but remained high in sedentary men $(10.5 \pm 1.4(\mathrm{IU} / \mathrm{gHb}) \mathrm{VS} 10.1 \pm 1.1$ (IU/gHb), for active and sedentary men, respectively; $\mathrm{P} \leq 0.05$ ). Also, G6PD levels showed a significant elevation 24 hours after long-duration exercise in the active men compared with the sedentary men $(11.8 \pm 2.5(\mathrm{IU} / \mathrm{gHb})$ VS $9.5 \pm 1.5(\mathrm{IU} / \mathrm{gHb})$, for active and sedentary men, respectively; $\mathrm{P} \leq 0.05$ ). As table 3 shows, G6PD activity was found to have increased immediately and 2 hours after long-duration exercise in sedentary men. The increase immediately and 2 hours after long-duration exercise in G6PD activity did not attain statistical significance $(\mathrm{P} \leq 0.05)$.

As it was expected, G6PD activity was not significantly increased immediately after long-duration in active men. G6PD levels returned at rest 2 hours after long-duration exercise as compared with immediately after long-duration in active men. Also, G6PD levels showed a significant elevation 24 hours after long-duration exercise in active men $(\mathrm{P}<0.05)$. The results of repeated measurements test are presented in table 4 .

Table 2. Results of independent sample t-test on characteristics of active and sedentary men (mean $\pm \mathrm{SD}$ )

\begin{tabular}{|c|c|c|c|}
\hline & Sedentary men (A) & Active men (B) & Significant Level $(\mathrm{P} \leq 0.05)$ \\
\hline Age (yr) & $19.5 \pm .084$ & $20.20 \pm 1.47$ & .210 \\
\hline Weight (kg) & $72.01 \pm 12.30$ & $66.28 \pm 6.77$ & .213 \\
\hline Height $(\mathrm{cm})$ & $176.2 \pm 7.47$ & $175.01 \pm 3.55$ & .652 \\
\hline BMI $\left(\mathrm{kg} / \mathrm{m}^{2}\right)$ & $23.2 \pm 3.67$ & $21.58 \pm 1.93$ & .234 \\
\hline Body fat (\%) & $8.8 \pm 5.75$ & $5.72 \pm 2.33$ & .126 \\
\hline $\mathrm{Vo}_{2 \max }\left(\mathrm{ml} \mathrm{kg}^{-1} \mathrm{~min}^{-1}\right)$ & $42.03 \pm 5.03$ & $58.51 \pm 5.09$ & .000 \\
\hline
\end{tabular}


Table 3. Results of independent sample t-test on G6PD activity of active and sedentary men (mean \pm SD)

\begin{tabular}{cccccc}
\hline \multirow{2}{*}{ Time } & \multicolumn{2}{c}{ G6PD activity (IU/gHb) } & & & \\
& Sedentary men (A) & Active men (B) & df & $t$ & Significant Level \\
\hline Pre-exercise & $9.49 \pm 1.04$ & $10.51 \pm 1.20$ & 18 & -2.018 & 0.059 \\
Post-exercise & $9.89 \pm 1.10$ & $11.62 \pm 2.70$ & 18 & -1.872 & 0.086 \\
2hours after exercise & $10.05 \pm 1.14$ & $10.51 \pm 1.39$ & 18 & -0.808 & 0.430 \\
24hours after exercise & $9.50 \pm 1.54$ & $11.75 \pm 2.48^{*}$ & 15.031 & -2.431 & 0.028 \\
\hline
\end{tabular}

Table 4. Results of repeated measurements test for G6PD activity changes in different times within each group (mean \pm SD)

\begin{tabular}{ccccccc}
\hline & \multicolumn{2}{c}{ Different Mean } & \multicolumn{2}{c}{ Standard Error } & \multicolumn{2}{c}{ Significant Level } \\
\hline Time & Sedentary & Active & Sedentary & Active & Sedentary & Active \\
Pre-Post exercise & -0.400 & -1.110 & 0.297 & 0.792 & 0.210 & 0.195 \\
Pre-2hours after exercise & -0.560 & $1.77 \mathrm{E}-15$ & 0.354 & 0.622 & 0.148 & 1.000 \\
Pre-24hours after exercise & -0.010 & -1.240 & 0.509 & 0.924 & 0.985 & 0.212 \\
Post-2hours after exercise & -0.160 & 1.110 & 0.389 & 0.893 & 0.690 & 0.245 \\
Post-24hours after exercise & 0.390 & -1.130 & 0.366 & 0.809 & 0.314 & 0.876 \\
2hours-24hours after exercise & 0.550 & -1.240 & 0.330 & 0.691 & 0.130 & 0.106 \\
\hline
\end{tabular}

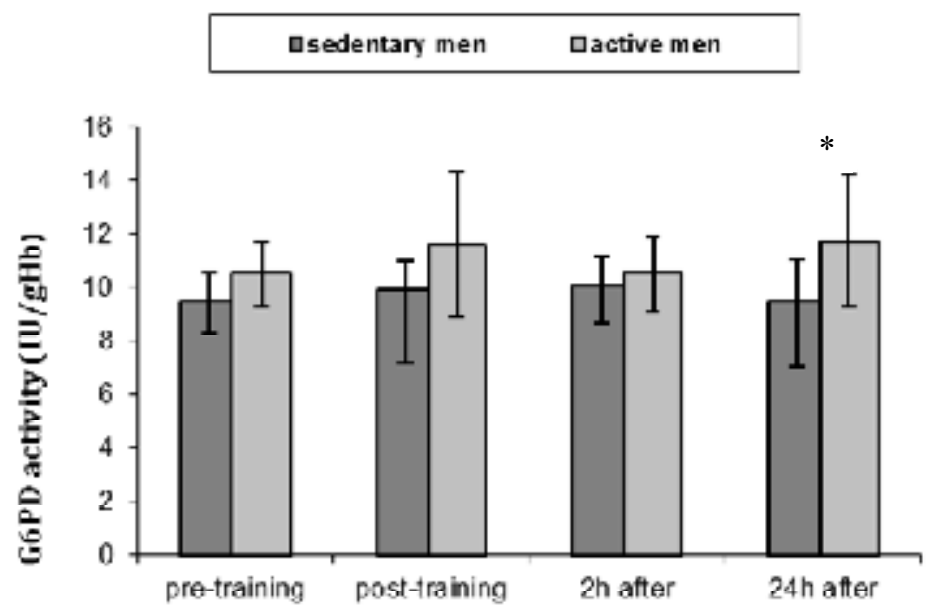

Figure 2. The effect of exercise on G6PD activity in active and sedentary men (mean \pm SD). *Significantly different from respective value in active men.

\section{Discussion}

Physical exercise is marked by an increase in oxygen consumption by the entire body, especially by the muscle tissue. This increase in oxygen consumption is associated with an increase in generation of reactive oxygen species (ROS) (Gomes, Silva, \& Oliveira, 2012). The high production of ROS might be responsible for a series of physiological and biochemical changes which happen at the time of exercise. It has been reported that exhausting exercise leads to a decrease in the levels of antioxidant as well as an increase in the marker of lipid peroxidation in blood lipoprotein and target tissues (Powers \& Jackson, 2008).In addition, the increase in cellular metabolism and hemoglobin turnover, as a result of physical exercise, may increase ROS generation by the erythrocytes, which primarily depend on the pentose phosphate pathway as their only sources of NADPH. Yet, erythrocytes, due to their highly limited biosynthetic capacity and low repair mechanism, whenever are exposed to extraordinary oxidative stress may acquire physical or molecular alteration. Indeed, ROS can modify the chemical cell membranes by altering the composition, distribution and packaging of their lipids (Groussard et al., 2003; Machefer et al., 2004). This lead to construct Ural modification of the cell membrane demonstrated by a decrease in membrane fluidity, which could alter the activity of certain membrane proteins and may inhibit accelerated senescence or even premature removal (Jamurtas et al., 2006; Tsakiris, Reclos, et al., 2006).

Our hypothesis suggested that exercise would lead to higher G6PD activity in active men at rest and after exercise. However, we found that G6PD activity was not significantly higher in active men compared with sedentary men at baseline. We realized that only in some of the investigations regular exercise affected G6PD activity. For instance, James et al reported that after performing a 12 week aerobic training program, G6PD activity increased significantly compared to baseline value in Down syndrome individuals (James et al., 2004). Also, in studies by Spodaryk et al carried out on male Wistar rats which had been subjected to running in an electric rotating drum, showed that training 
induced an improvement of G6PD activity. Each training session lasted thirty days (Spodaryk, Szyguła, Dabrowski, \& Miszta, 1985). Thus, we realized that physical activity had an effect on G6PD activity, as reported by Ordonez et al, Spodaryk et al and other researchers (James et al., 2004; Spodaryk et al., 1985). In this way, we generalized that G6PD activity is higher in active men at baseline. This agrees with our findings. The activity of antioxidant enzymes depends on the rate of radical generation; for example, an increase in superoxide formation stimulates superoxide dismutase expression and activity. Therefore, it is generally accepted that regular exercise increases the activity of the antioxidant enzyme system (Berzosa et al., 2011; Gomes et al., 2012; Radak, Chung, \& Goto, 2008).

G6PD activity was found to have increased in both groups immediately after exercise but was not significantly different between the groups $(\mathrm{P} \leq 0.05)$, as reported (see table 3) by (Ohno, Yahata, Sato, Yamamura, \& Taniguchi, 1988; Spodaryk, 2001; Vesovic, Borjanovic, Markovic, \& Vidakovic, 2001)1. The aim of Vesovic et al and Spodaryk et al studies was to determine the activity of antioxidant enzymes in erythrocytes during exhausting short-duration exercise. Also, the aim of Ohno et al study was to observe the effect of short-duration physical exercise on free radical scavenging system enzymes of the erythrocytes. Schulpis et al, Tsakiris et al and Georgakouli et al have not obtained the same findings (Georgakouli et al., 2013; Schulpis et al., 2006; Tsakiris, Parthimos, Parthimos, Tsakiris, \& Schulpis, 2006). In Schulpis et al study, G6PD activity was found remarkably decreased pre versus post ultra-distance foot race "Spartathlon". This discrepancy may be due to the difference in the intensity and duration of the exercise and age of the participants. Schulpis et al used individuals median aged 36.5 year, whereas other studies used teenagers. In all the studies by Tsakiris et al, Schulpis et al and Brady et al, supplementation of antioxidants was used. Studies indicate that supplementation of antioxidants in the diet has protective impact on oxidative stress induced by exercise (Georgakouli et al., 2013; Jamurtas et al., 2006). Because G6PD enzyme is a supplementation of antioxidants, it seems very probable that supplementations of antioxidants lead to G6PD activity reduction. This idea is supported by the positive correlation of G6PD activity with supplementation of antioxidants, as found in studies (Brady, Brady, \& Ullrey, 1979; Schulpis et al., 2006; Tsakiris, Reclos, et al., 2006; Tsakiris, Schulpis, Marinou, \& Behrakis, 2004).

There is not agreement among researchers about production of antioxidant enzymes as a result of exercise (Banerjee, Mandal, Chanda, \& Chakraborti, 2003; Sen, 1995; Urso \& Clarkson, 2003). At the time of physical activity, it could be assumed that tissues are protected by a positive regulation of antioxidant. In humans, a greater antioxidant enzyme activity was reported to correlate with $\mathrm{VO}_{2 \max }$, and trained athletes were shown to have higher antioxidant enzymes activity in skeletal muscles (Theodorou et al., 2010). According to these statements, G6PD activity immediately after long-duration exercise in active subjects could be explained. After 2 hours, G6PD activity was higher in active men than in sedentary men. It should be noted that in available investigations, there is no blood sample obtained from the 2 hours post-exercise phase. Increase in G6PD activity after 2 hours might be due to increased oxidative stress during exercise.

After 24 hours of exercise, G6PD activity was remarkably higher in active men compared with sedentary men. Within this context, it must be mentioned that in previous studies there is no blood sample obtained for 24 hours after exercise. As reported in humans, a higher antioxidant enzyme activity was reported to correlate with $\mathrm{VO}_{2 \max }$ (Theodorou et al., 2010). Thus, as a long-term strategy, the cells in active individuals might trigger de novo synthesis of antioxidant enzymes in order to deal with stress (Berzosa et al., 2011; Gomes et al., 2012; Radak et al., 2008). The mechanisms involved in the increased antioxidant enzyme activity during exercise are still unknown. Although evidence is emerging that mammalian tissues can up-regulate gene expression of antioxidant enzymes in response to acute oxidative stress, the signal transduction pathway is still unknown (Gomes et al., 2012; Radak et al., 2008).

In sedentary men, G6PD activity amount was increased immediately and 2 hours after exercise. As expected, this increase in G6PD is primarily due to increased number of free radicals. In contrast, 24 hours after exercise, G6PD level returns at rest, but is not significantly different from other times. Because G6PD enzyme is an antioxidant, increasing the levels of antioxidant enzymes by exercise training would prevent or attenuate damaging lipid peroxidation during exercise.

In active men, G6PD activity amount was increased immediately after exercise but returned at rest 2 hours after exercise in this group. Interestingly, G6PD activity amount was remarkably increased 24 hours after exercise in this group. Growing evidence suggests that physical endurance training up-regulates the level of antioxidant enzymes in tissues actively involved in exercise (Fisher, 2010; Gonchar \& Mankovska, 2010; Ji, Gomez-Cabrera, \& Vina, 2009; Margaritis, Rousseau, Marini, \& Chopard, 2009; Vina, Borras, Gomez-Cabrera, \& Orr, 2006), and that this may decrease susceptibility to oxidative stress. In other words, the observed increase in G6PD activity 24 hours after exercise in active men may also be due to compatibility with exercise.

\section{Conclusion}

In this regard, it can be concluded that progressive aerobic exercise may be an effective factor affecting the levels of G6PD significantly, and as a home message it is useful for controlling the hemolytic anemia among sedentary population.

\section{Acknowledgements}

This study was supported in part by the University of Mohaghegh Ardabili in Iran. Many thanks are expressed to Dr. Jafar Zadeh, Dr. Shokrabadi and Ehsan Najafi for their significant assistance.

\section{References}

Ainoon, O, Yu, YH, Amir Muhriz, AL, Boo, NY, Cheong, SK, \& Hamidah, NH. (2003). Glucose-6-phosphate dehydrogenase (G6PD) variants in Malaysian Malays. Human mutation, 21(1), 101-101. 
Allahverdiyev, Adil M, Bagirova, Malahat, Elcicek, Serhat, Koc, Rabia Cakir, Ates, Sezen Canim, Baydar, Serap Yesilkir, ... Oztel, Olga Nehir. (2012). Glucose-6-phosphate dehydrogenase deficiency and malaria: a method to detect primaquine-induced hemolysis in vitro. Dehydrogenases, Prof. Rosa Angela Canuto (Ed.).

Banerjee, Alok K, Mandal, Amritlal, Chanda, Dipanjan, \& Chakraborti, Sajal. (2003). Oxidant, antioxidant and physical exercise. Molecular and cellular biochemistry, 253(1-2), 307-312.

Berzosa, C, Cebrian, I, Fuentes-Broto, L, Gomez-Trullen, E, Piedrafita, E, Martinez-Ballarin, E, . . Garcia, JJ. (2011). Acute exercise increases plasma total antioxidant status and antioxidant enzyme activities in untrained men. BioMed Research International, 2011.

Bloomer, Richard J, Goldfarb, Allan H, Wideman, Laurie, Mckenzie, Michael J, \& Consitt, Leslie A. (2005). Effects of acute aerobic and anaerobic exercise on blood markers of oxidative stress. The Journal of Strength \& Conditioning Research, 19(2), 276-285.

Bommareddy, Rajesh Reddy, Chen, Zhen, Rappert, Sugima, \& Zeng, An-Ping. (2014). A de novo NADPH generation pathway for improving lysine production of Corynebacterium glutamicum by rational design of the coenzyme specificity of glyceraldehyde 3-phosphate dehydrogenase. Metabolic Engineering, 25(0), 30-37. doi: http://dx.doi.org/10.1016/j.ymben.2014.06.005

Brady, Paul S, Brady, Linda J, \& Ullrey, Duane E. (1979). Selenium, vitamin E and the response to swimming stress in the rat. The Journal of nutrition, 109(6), 1103-1109.

Fisher-Wellman, Kelsey, \& Bloomer, Richard J. (2009). Acute exercise and oxidative stress: a 30 year history. Dynamic Medicine, 8(1), 1.

Fisher, Gordon. (2010). Oxidative Stress and Antioxidant Defenses in Lymphocytes Following High Intensity Interval Training. Auburn University.

Georgakouli, Kalliopi, Deli, Chariklia K, Zalavras, Athanasios, Fatouros, Ioannis G, Kouretas, Dimitrios, Koutedakis, Yiannis, \& Jamurtas, Athanasios Z. (2013). $\alpha$-Lipoic acid supplementation up-regulates antioxidant capacity in adults with G6PD deficiency. Food and Chemical Toxicology, 61, 69-73.

Gomes, Elisa Couto, Silva, Albená Nunes, \& Oliveira, Marta Rubino de. (2012). Oxidants, antioxidants, and the beneficial roles of exercise-induced production of reactive species. Oxidative medicine and cellular longevity, 2012.

Gonchar, O, \& Mankovska, I. (2010). Antioxidant system in adaptation to intermittent hypoxia. Journal of Biological Sciences, 10(6), 545-554.

Groussard, C, Rannou-Bekono, F, Machefer, G, Chevanne, M, Vincent, S, Sergent, O, . . Gratas-Delamarche, A. (2003). Changes in blood lipid peroxidation markers and antioxidants after a single sprint anaerobic exercise. European journal of applied physiology, 89(1), 14-20.

Huang, Yang-Yang, Huang, Ching-Shui, Yang, Sien-Sing, Lin, Min-Shung, Huang, May-Jen, \& Huang, Ching-Shan. (2005). Effects of variant UDP-glucuronosyltransferase $1 \mathrm{~A} 1$ gene, glucose-6-phosphate dehydrogenase deficiency and thalassemia on cholelithiasis. World Journal of Gastroenterology, 11(36), 5710.

James, S Jill, Cutler, Paul, Melnyk, Stepan, Jernigan, Stefanie, Janak, Laurette, Gaylor, David W, \& Neubrander, James A. (2004). Metabolic biomarkers of increased oxidative stress and impaired methylation capacity in children with autism. The American journal of clinical nutrition, 80(6), 1611-1617.

Jamurtas, Athanasios Z, Fatouros, Ioannis G, Koukosias, Nikos, Manthou, Eirini, Tofas, Trifon, Yfanti, Christina, ... Koutedakis, Yiannis. (2006). Effect of exercise on oxidative stress in individuals with glucose-6-phosphate dehydrogenase deficiency. in vivo, 20(6B), 875-880.

Ji, Li L, Gomez-Cabrera, Maria-Carmen, \& Vina, Jose. (2009). Role of free radicals and antioxidant signaling in skeletal muscle health and pathology. Infectious Disorders-Drug Targets (Formerly Current Drug Targets-Infectious Disorders), 9(4), 428-444.

Machefer, Guillaume, Groussard, Carole, Rannou-Bekono, Françoise, Zouhal, Hassane, Faure, Henry, Vincent, Sophie, . . Gratas-Delamarche, Arlette. (2004). Extreme running competition decreases blood antioxidant defense capacity. Journal of the American College of Nutrition, 23(4), 358-364.

Margaritis, I, Rousseau, AS, Marini, JF, \& Chopard, A. (2009). Does antioxidant system adaptive response alleviate related oxidative damage with long term bed rest? Clinical biochemistry, 42(4), 371-379.

Masella, Roberta, Di Benedetto, Roberta, Varì, Rosaria, Filesi, Carmela, \& Giovannini, Claudio. (2005). Novel mechanisms of natural antioxidant compounds in biological systems: involvement of glutathione and glutathionerelated enzymes. The Journal of nutritional biochemistry, 16(10), 577-586.

Nazıroğlu, Mustafa. (2009). Role of selenium on calcium signaling and oxidative stress-induced molecular pathways in epilepsy. Neurochemical research, 34(12), 2181-2191.

Niess, Andreas Michael, \& Simon, Perikles. (2007). Response and adaptation of skeletal muscle to exercise-the role of reactive oxygen species. Front Biosci, 12, 4826-4838.

Nikolaidis, Michalis G, Jamurtas, Athanasios Z, Paschalis, Vassilis, Kostaropoulos, Iason A, Kladi-Skandali, Athina, Balamitsi, Vera, ... Kouretas, Dimitris. (2006). Exercise-induced oxidative stress in G6PD-deficient individuals. Medicine and science in sports and exercise, 38(8), 1443-1450.

Ohno, H, Yahata, T, Sato, Y, Yamamura, K, \& Taniguchi, N. (1988). Physical training and fasting erythrocyte activities of free radical scavenging enzyme systems in sedentary men. European journal of applied physiology and occupational physiology, 57(2), 173-176.

Oztasan, Nuray, Taysi, Seyithan, Gumustekin, Kenan, Altinkaynak, Konca, Aktas, Omer, Timur, Handan, ... Akcay, Fatih. (2004). Endurance training attenuates exercise-induced oxidative stress in erythrocytes in rat. European journal of applied physiology, 91(5-6), 622-627.

Petrat, Frank, Pindiur, Stanislaw, Kirsch, Michael, \& de Groot, Herbert. (2003). NAD (P) H, a primary target of $1 \mathrm{O} 2$ in mitochondria of intact cells. Journal of Biological Chemistry, 278(5), 3298-3307. 
Powers, Scott K, \& Jackson, Malcolm J. (2008). Exercise-induced oxidative stress: cellular mechanisms and impact on muscle force production. Physiological reviews, 88(4), 1243-1276.

Radak, Zsolt, Chung, Hae Young, \& Goto, Sataro. (2008). Systemic adaptation to oxidative challenge induced by regular exercise. Free Radical Biology and Medicine, 44(2), 153-159.

Sahlin, Kent, Shabalina, Irina G, Mattsson, C Mikael, Bakkman, Linda, Fernström, Maria, Rozhdestvenskaya, Zinaida, . . Tonkonogi, Michail. (2010). Ultraendurance exercise increases the production of reactive oxygen species in isolated mitochondria from human skeletal muscle. Journal of applied physiology, 108(4), 780-787.

Schafer, Freya Q, \& Buettner, Garry R. (2001). Redox environment of the cell as viewed through the redox state of the glutathione disulfide/glutathione couple. Free Radical Biology and Medicine, 30(11), 1191-1212.

Schulpis, Kleopatra H, Reclos, George J, Parthimos, Theodore, Parthimos, Nickolaos, Gavriilidis, Andreas, \& Tsakiris, Stylianos. (2006). L-cysteine supplementation protects the erythrocyte glucose-6-phosphate dehydrogenase activity from reduction induced by forced training. Clinical biochemistry, 39(10), 1002-1006.

Sen, Chandan K. (1995). Oxidants and antioxidants in exercise. Journal of Applied Physiology, 79(3), 675-686.

Shariat, Ardalan, Kargarfard, Mehdi, Tamrin, Shamsul Bahri Mohd, Danaee, Mahmoud, \& Karimi, Hossein. (2014). Strength-training and biological rhythm of male sex hormone among judoists. Biological Rhythm Research(ahead-ofprint), 1-7.

Spodaryk, Krzysztof. (2001). The influence of low-power laser energy on red blood cell metabolism and deformability. Clinical hemorheology and microcirculation, 25(3), 145-151.

Spodaryk, Krzysztof, Szyguła, Zbigniew, Dabrowski, Zbigniew, \& Miszta, Helena. (1985). The activity of erythrocyte enzymes in rats subjected to running exercises. European journal of applied physiology and occupational physiology, 54(5), 533-537.

Theodorou, Anastasios A, Nikolaidis, Michalis G, Paschalis, Vassilis, Sakellariou, Georgios K, Fatouros, Ioannis G, Koutedakis, Yiannis, \& Jamurtas, Athanasios Z. (2010). Comparison between glucose-6-phosphate dehydrogenasedeficient and normal individuals after eccentric exercise. Med Sci Sports Exerc, 42(6), 1113-1121.

Tsakiris, Stylianos, Parthimos, Theodore, Parthimos, Nickolaos, Tsakiris, Theodore, \& Schulpis, Kleopatra H. (2006). The beneficial effect of L-cysteine supplementation on DNA oxidation induced by forced training. Pharmacological research, 53(4), 386-390.

Tsakiris, Stylianos, Reclos, George J, Parthimos, Theodore, Tsakiris, Theodore, Parthimos, Nickolaos, \& Schulpis, Kleopatra H. (2006). $\alpha$-Tocopherol supplementation restores the reduction of erythrocyte glucose-6-phosphate dehydrogenase activity induced by forced training. Pharmacological research, 54(5), 373-379.

Tsakiris, Stylianos, Schulpis, Kleopatra H, Marinou, Kyriakoula, \& Behrakis, Panagiotis. (2004). Protective effect of Lcysteine and glutathione on the modulated suckling rat brain $\mathrm{Na}+, \mathrm{K}+,-\mathrm{ATPase}$ and $\mathrm{Mg} 2+-\mathrm{ATPase}$ activities induced by the in vitro galactosaemia. Pharmacological research: the official journal of the Italian Pharmacological Society, 49(5), 475-479.

Urso, Maria L, \& Clarkson, Priscilla M. (2003). Oxidative stress, exercise, and antioxidant supplementation. Toxicology, 189(1), 41-54.

Vesovic, D, Borjanovic, S, Markovic, S, \& Vidakovic, A. (2001). Strenuous exercise and action of antioxidant enzymes. La Medicina del lavoro, 93(6), 540-550.

Vina, Jose, Borras, Consuelo, Gomez-Cabrera, Mari-Carmen, \& Orr, William C. (2006). Part of the Series: From Dietary Antioxidants to Regulators in Cellular Signalling and Gene ExpressionRole of reactive oxygen species and (phyto) oestrogens in the modulation of adaptive response to stress: Review. Free radical research, 40(2), 111-119.

Wamelink, MMC, Struys, EA, \& Jakobs, C. (2008). The biochemistry, metabolism and inherited defects of the pentose phosphate pathway: a review. Journal of inherited metabolic disease, 31(6), 703-717. 